\title{
A review of univariate and multivariate process capability indices
}

\author{
David de-Felipe • Ernest Benedito
}

Received: date / Accepted: date

\begin{abstract}
This paper offers a review of univariate and multivariate Process Capability Indices (PCIs). PCIs are used in the industry to quantify how well a process can meet customer requirements. Univariate PCIs describe the capability of one single product characteristic. Multivariate PCIs deal with the multivariate case in which the measures of all multiple product characteristics must be within specification limits to be conforming. When analyzing the capability of processes, decision makers of the industry may choose one PCI among all the PCIs existing in the literature, depending on different capability criteria. The aim of the review is to describe, cluster and discuss univariate and multivariate PCIs. This review may help researchers and decision makers to identify univariate and multivariate PCIs that can be used when monitoring univariate and multivariate production processes. On the one hand, the authors of this article suggest using PCIs obtained through the alternative definition for the $C_{p k}$ index when analyzing the capability of production processes, in which the estimation of the proportion of nonconforming parts is rated as crucial. On the other hand, all other multivariate PCIs presented in the literature can be applicable in capability analysis of production processes in which a direct relation to the proportion of nonconforming parts is not needed.
\end{abstract}

Keywords Process Capability Indices · Quality control · Process monitoring · Product reliability

\section{David de-Felipe}

Bayerische Motoren Werke AG, BMW AG, Munich, Germany

E-mail: David.de-Felipe@bmw.de

Ernest Benedito

Department of Management and Institute of Industrial and Control Engineering, Universitat Politècnica de Catalunya (UPC), Barcelona, Spain E-mail: ernest.benedito@upc.edu

\section{Introduction}

Today, organizations and enterprises carrying out their activities in competitive environments are continuously trying to achieve high levels of production and economy. Thus, optimization of production processes based on failure prevention, planing and control is on focus of study [1] [2] [3] [4] [5] [6]. The pioner of optimization models of production processes based on quality costs was Taguchi [7], whose principles and theories are accepted as reference models due to the revolution that they supposed to quality methods. In this context, Statistical Process Monitoring (SPM) is rated as a very important area of process control [8], to ensure economic productivity by detecting production failures such as collision or tool wear [9] and is contributing to saving costs in manufacturing [10].

Process Capability Indices (PCIs) are statistic indicators widely used in the industry in SPM to quantify how well a process can meet customer requirements by relating the variability of the measures of the product characteristics with the admissible one. A process is described as capable if it is able to produce products within the specification limits (SLs). Thus, PCIs are indicators of the goodness of the process related to the position and the variability of the measures within the SLs and are extensively accepted and used in the industry.

Originally, product quality was described considering only one product characteristic. Nowadays, due to the continuously improvement of production processes, product quality is analyzed by considering simultaneously several product characteristics. Thus, univariate production processes are tending to become multivariate production processes. 
In capability analysis, it is possible to distinguish between univariate and multivariate PCIs. On the one hand, univariate PCIs can be used, independently, to calculate the capability related to one single product characteristic. On the other hand, multivariate PCIs describe the capability of a multivariate process by taking into account all product characteristics in a global way.

In most companies, univariate PCIs are used to evaluate the capability of multivariate production processes by obtaining one univariate PCI for each product characteristic. The usage of this methodology can lead to misinterpretation because the capability of each product characteristic is analyzed independently, namely without taking into account its influence over the other product characteristics of the analyzed multivariate production process. In this context, when analyzing the capability of multivariate production processes, univariate PCIs need to be complemented with multivariate PCIs, which consider simultaneously all product characteristics. Multivariate PCIs have been introduced in the literature to describe the entire production variability derived from the multivariate case. Thanks to multivariate PCIs, the capability of a multivariate process with $v$ product characteristics can be summarized with one single index.

Taking a look at the available literature in this field, there seems to be an agreement in the scientific community to describe the capability of multivariate production processes by using multivariate PCIs. However, the industry has not adapted this kind of indicators when evaluating multivariate production processes. For this reason, in this article we summarize and discuss univariate and multivariate PCIs from the literature with the aim of giving a useful and practical review to the industry.

To date, several reviews of PCIs have been published in the literature. Kotz et al. [11] presented a review for the development of PCIs during the period 1992 to 2000. Wu, Pearn and Kotz [12] discussed the developments between years 2002 and 2008. Yum and Kim [13] presented a bibliography of literature between years 2000 and 2009. Although some PCIs have been presented recently in the literature to deal with dependent processes (e.g. Pan, Li and Chen [14] and Pan and Huan [15]) and linear and non-linear regression profiles (e.g. Ebadi and Amiri [16], Wang and Tamirat [17] and Guevara and Vargas [18] ), in this paper we focus on univariate and multivariate PCIs based on the traditional definition of capability introduced by Kane [19] in 1986. In order to normalize the nomenclature of all the PCIs presented in this paper, each index will be introduced using the same nomenclature criterion even

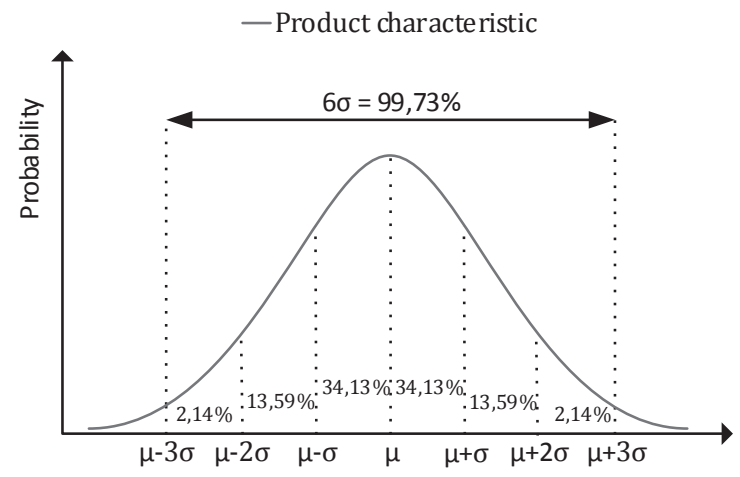

Fig. 1 Width of normal distributed measures of a product characteristic.

though the authors used a different nomenclature at submission time.

The purpose of this paper is to offer a review of process capability indices with two main objectives: First to describe the univariate and multivariate PCIs existing in the literature; and second to cluster and discuss them critically. For this reason, our review may help practitioners and researchers to have an overview of the existing work related to this topic. The rest of the paper has the following structure: In section 2, univariate PCIs existing in the literature are introduced. In section 3, multivariate PCIs existing in the literature are presented. In section 4, all univariate and multivariate PCIs presented in this review are clustered and discussed. The survey concludes in section 5 .

\section{Univariate Process Capability Indices}

Univariate PCIs are statistic indicators used to quantify the goodness of a process by relating the variability of the measures of a single product characteristic with the admissible one.

It is accepted that the measures of the product characteristics obtained in the quality tests follow a normal distribution in most of production processes (Montgomery [20]). Thus, their width (variability) can be described with the variance $\left(\sigma^{2}\right)$ of the product characteristic distribution, which is the expected value of the squared deviation from the mean of the data. About $99.73 \%$ of the values drawn from a normal distribution are within six sigma $(\sigma)$ away from the mean (see figure 1).

Sullivan [21] introduced in the literature the univariate PCIs $C_{p}, C P U, C P L, k$ and $C_{p k}$. Kane [19] introduced various applications of these indices and dis- 
cussed along with statistical sampling considerations to evaluate production processes.

Henceforth two concepts are introduced: the process region $(\mathrm{PR})$ and the specification region $(\mathrm{SR})$. These two regions will help the reader to understand the differences between the presented PCIs. The definition of both regions will be adapted to the multivariate case in the next section and will be also used to explain the multivariate PCIs.

The PR is defined as the interval that includes 99.73 $\%$ of values drawn from a normal distribution and which is centered on the mean value of the measured product characteristic. Thus, in the univariate case, the lowest point of the $\mathrm{PR}$ is placed at $\mu-3 \sigma$; and the highest point at $\mu+3 \sigma$. The $\mathrm{SR}$ is defined as the interval limited by the lower specification limit (LSL) and the upper specification limit (USL).

\subsection{The $C_{p}$ index}

The $C_{p}$ index is an univariate PCI that shows if the analyzed process can be capable. With this index, the width of $\mathrm{SR}(U S L-L S L)$ and the width of the PR $(6 \sigma)$ are compared. Figure 2 shows both regions. The $C_{p}$ index can be calculated with this formula:

$C_{p}=(U S L-L S L) / 6 \sigma$

Unfortunately, obtaining measures of the whole population is usually difficult, if not impossible. Thus, many times it is not possible to describe the PR with its real variance $\left(\sigma^{2}\right)$. For this reason, many times, the variance is estimated with the standard deviation $(s)$ of a data sample $\left(s=\sqrt{\sigma^{2}}\right)$.

According to Tsai and Chen [22] it is possible to distinguish between several levels of capability when using the $C_{p}$ index: super excellent for $C_{p}$ values higher than 2.00; excellent for values between $1.67 \leq C_{p}<2.00$; satisfactory for values between $1.33 \leq C_{p}<1.67$; capable for values between $1.00 \leq C_{p}<1.33$; inadequate for values between $0.67 \leq C_{p}<1.00$; and poor for $C_{p}$ values smaller than 0.67 .

The $C_{p}$ index only takes into account the width of the PR, but it does not consider its position within the $\mathrm{SR}$. If the PR is not centered on the SR, it would be possible to have a substantial percentage of products with characteristics outside the SL although the $C_{p}$ value is high. In order to solve this problem, the CPU, CPL, $k$ and $C_{p k}$ indices were also introduced.

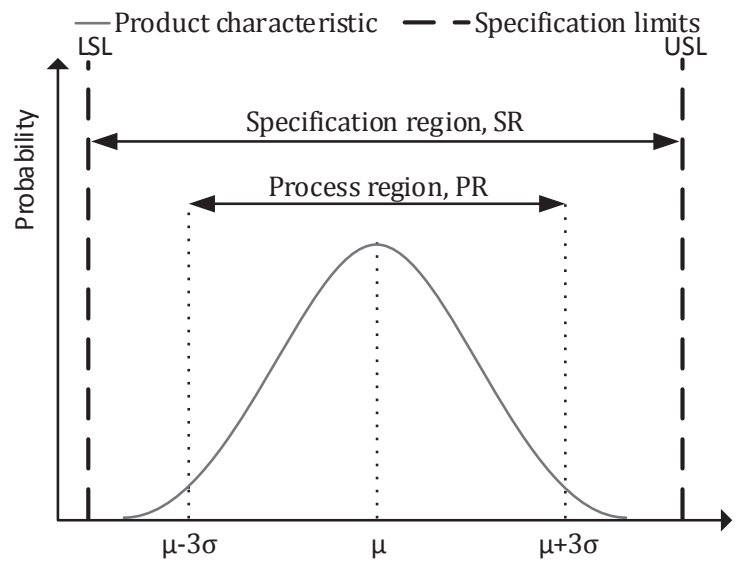

Fig. 2 Process and specification regions.

\subsection{The $C P U$ and the $C P L$ indices}

The $C P U$ index describes the relation between the upper half of the $\mathrm{SR}(U S L-\mu)$ and half $\operatorname{PR}(3 \sigma)$. The $C P L$ index describes the relation between the lower half of the SR $(\mu-L S L)$ and half PR. Hereafter, the formula of both indices is introduced:

$$
\begin{aligned}
& C P U=(U S L-\mu) / 3 \sigma \\
& C P L=(\mu-L S L) / 3 \sigma
\end{aligned}
$$

As in the case of the $C_{p}$ index, the variance of the population can be estimated with the standard deviation of a data sample. In this case, the mean of the population $(\mu)$ can be estimated with the mean value $(\bar{x})$ of a data sample. These estimations are valid for each index presented henceforth.

\subsection{The $C_{p k}$ and the $k$ indices}

The $C_{p k}$ index considers the minimal distance between the midpoint of the PR and its closer SL. This index is the minimal value between the $C P U$ and the $C P L$ indices. This index is widely used in the industry because of their easy usage and interpretation. Hereafter, the formula of the $C_{p k}$ index is introduced:

$$
C_{p k}=\min \{C P L, C P U\}
$$

The $k$ index describes the distance between the target of the product characteristic $(T)$ and the mean value of the product characteristic:

$k=\frac{|T-\mu|}{\frac{U S L-L S L}{2}}$ 


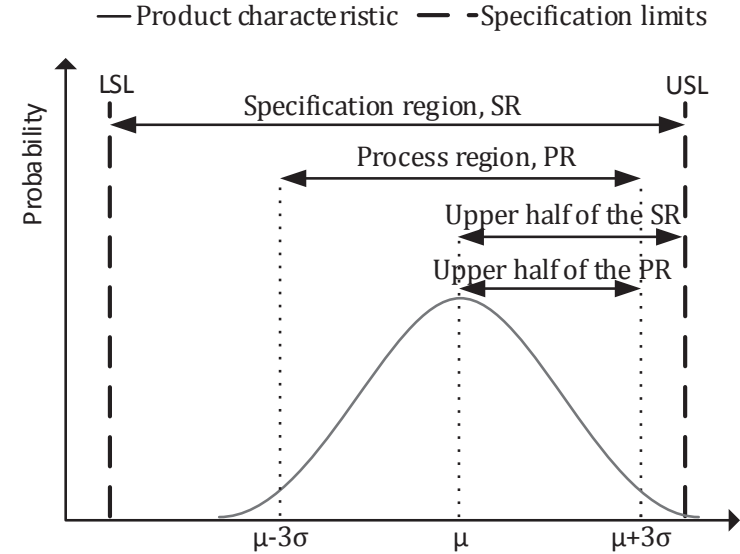

Fig. 3 Relation between the upper half of the SR and the upper half of the PR.

When the target value is the midpoint of the SR, the $C_{p k}$ and the $\mathrm{k}$ indices are related by the following expression:

$C_{p k}=C_{p}(1-k)$

If the mean value of the product characteristic is exactly in the middle of the SR, the $C_{p k}$ and the $C_{p}$ indices have the same value. If the $C_{p k}$ index is bigger than 1 , the process is defined as capable. However, many companies are specifying $C_{p k}$ goals of 1.33 (Bothe [23]). In figure 3 the upper half of the PR as well of the SR are represented.

\subsection{The $C_{p m}$ index}

Chan, Cheng and Spiring [24] introduced the $C_{p m}$ index (8). This index also considers the possibility that the target value is not the middle point of the SR. For this reason, in Chan, Cheng and Spiring [24] a modification of the PR is proposed. The modified PR is the interval $\left[T-3 \sigma^{\prime}, T+3 \sigma^{\prime}\right]$ where:

$\sigma^{\prime}=\sqrt{\frac{\sum_{i}^{n}\left(x_{i}-T\right)^{2}}{n-1}}$

The modified PR includes $99.73 \%$ of values drawn from a normal distribution which is centered on the target value and has an estimated variance $\left(\sigma^{\prime 2}\right)$ that is calculated by taking into account the distance between the product characteristic and the target value.

$C_{p m}=\frac{\min (U S L-T, T-L S L)}{3 \sigma^{\prime}}$
- Product characteristic - - Specification limits

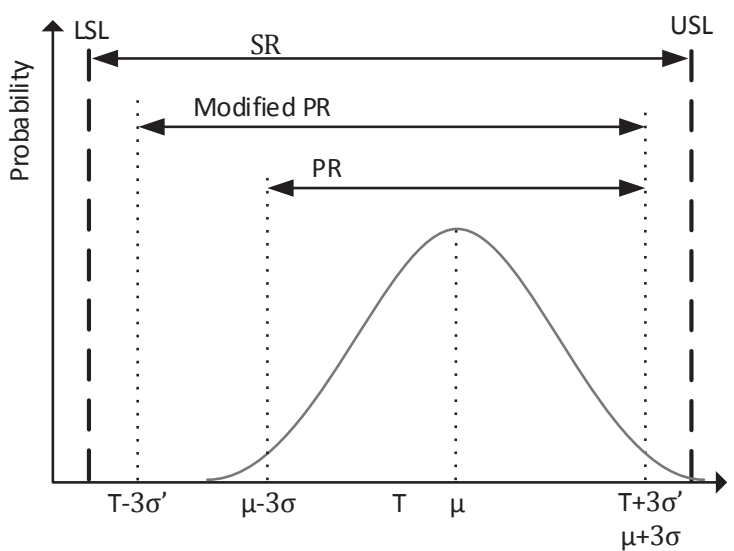

Fig. 4 PR and modified PR according Chan. Cheng and Spiring [24].

Figure 4 shows an example of the original and modified PR as well as the SR. In the case of this figure, $\mu+3 \sigma^{\prime}$ and $T+3 \sigma$ overlap.

\subsection{The $C_{p m k}$ index}

Pearn, Kotz and Johnson [25] introduced the $C_{p m k}$ index (9). It is a combination of the $C_{p k}$ and the $C_{p m}$ indices. This index considers the position of the mean value of the product characteristic within the $S R$ and also supposes that the target value is not centered on the SR.

$C_{p m k}=\frac{\min (U S L-\mu, \mu-L S L)}{3 \sigma^{\prime}}$

The modified PR' used in the $C_{p m k}$ index is the interval $\left[\mu-3 \sigma^{\prime}, \mu+3 \sigma^{\prime}\right]$. It has the same width as the modified PR in the $C_{p m}$ index but it is centered on the mean value of the measures as the PR in the $C_{p k}$ index. Figure 5 helps to understand the differences in the value of the $C_{p k}, C_{p m}$ and $C_{p m k}$ indices, using the same example as in figure 4 . It is easy to see that not only the width of the modified PR but also its position have an influence on the value of the index, and thus, in capability analysis. In the case of figure 5, the upper half of the SR is smaller than the upper half of the modified PR'. Thus, the $C_{p m k}$ index is smaller than one and, consequently, the process is not capable. However, the $C_{p m}$ and the $C_{p k}$ indices are higher than 1 and, consequently, regarding these indices the process is capable. 


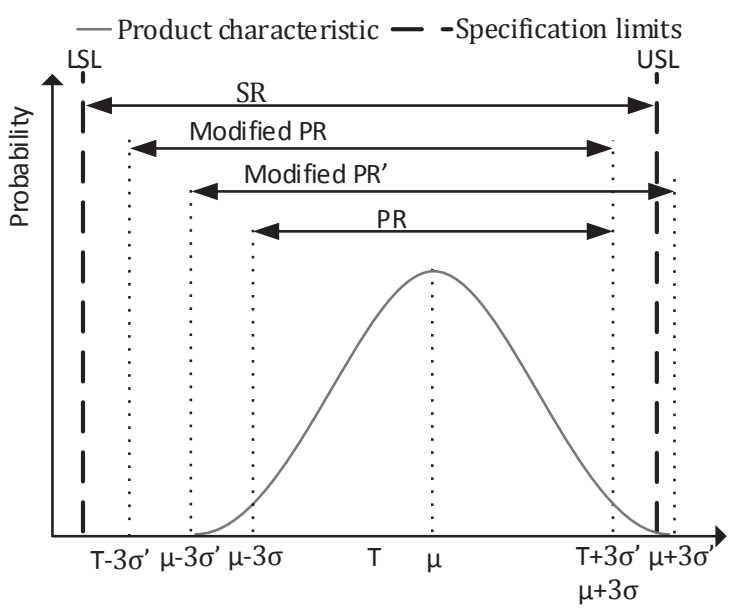

Fig. 5 Modified PR' according Pearn, Kotz and Johnson [25].

\subsection{Other univariate PCIs}

Hereafter, we introduce other univariate PCIs from the literature. The following PCIs provide a different point of view when analyzing the capability of production processes and thus, the inclusion of these indices gives value added to this literature review.

\subsubsection{The maximal allowable standard deviation}

González and Sánchez [26] introduced the $C_{n}$ index. Considering that the measured data follows a normal distribution, $X \sim N(\mu, \sigma)$, with this index it is possible to compare the standard deviation of the measured product characteristic $(s)$ with the maximal allowable one $\left(s_{\max }\right)$. The maximal allowable standard deviation is the maximal standard deviation that the measured product characteristic could have in order to verify that the probability that a measure of this product characteristic is located inside the $\mathrm{SR}$ is the minimal acceptable one $\left(P_{\min }\right): P\left(L S L \leq X \sim N\left(\mu, s_{\max }\right) \leq U S L\right)=$ $P_{\min }$. The $C_{n}$ index can be calculated with the next formula:

$C_{n}=\frac{s_{\max }}{s}$

As it can be seen, a process is capable $\left(C_{n}\right.$ values higher than 1) if the variability of the measured product characteristic $(s)$ is smaller than the maximal allowable one $\left(s_{\max }\right)$.

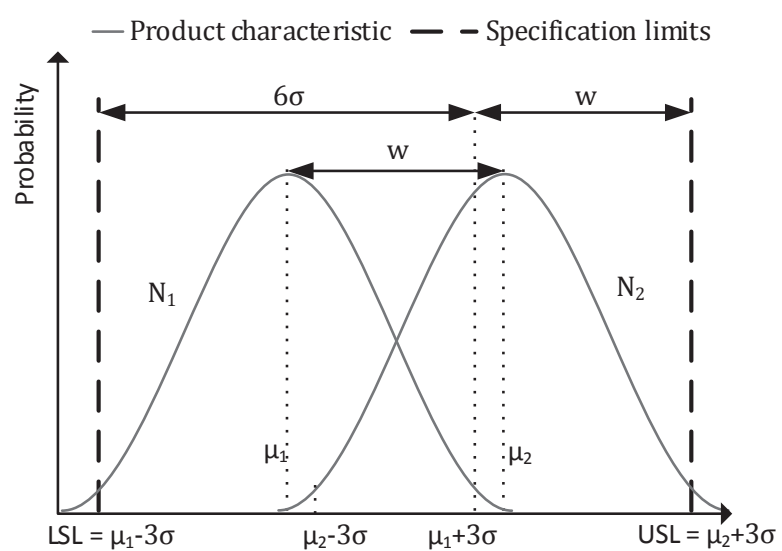

Fig. 6 Window of opportunity as stated in Veevers [27].

\subsubsection{The window of opportunity}

Veevers [27] introduced the concept of Viability $\left(V_{r}\right)$ of a process by describing its window of opportunity $(w)$. The window of opportunity is the interval in which the center of the PR can be placed with the condition that the PR is inside the SR. Taking it into account, the Viability is defined as:

$V_{r}=\frac{w}{U S L-L S L}$

If the measures of the product characteristic follow a normal distribution, the window of opportunity can be approximated to:

$w=U S L-L S L-6 \sigma$

Imagine that we want to analyze a process that generates outputs whose product characteristics follow a normal distribution, $X \sim N(\mu, \sigma)$. In order to define the window of opportunity we have to define two auxiliary processes with the same $\sigma$ value and centered on the extreme values of the window of opportunity.

Figure 6 shows both auxiliary processes: on the left side there is a process $N_{1}\left(\mu_{1}, \sigma\right)$, whose $0.135 \%$ of the data is under the $L S L\left(\mu_{1}-3 \sigma=L S L\right)$; on the right side there is a process $N_{2}\left(\mu_{2}, \sigma\right)$, whose $0.135 \%$ of the data is over the $U S L\left(\mu_{2}+3 \sigma=L S L\right)$. The distance between $\mu_{1}$ and $\mu_{2}$ is the window of opportunity.

As it can be seen, a process is capable if the mean value of the measured data is within the window of opportunity. 


\subsubsection{Loss-based PCIs}

Another way to tackle the problem of the capability analysis is to describe the goodness of a process by estimating its loss. The loss of a process is defined as the cost arising from the production of nonconforming parts. In this context, Johnson [28] suggested using another dimensionless indicator to describe the capability of production processes and defined $L_{e}$ as the ratio of the expected quadratic loss, which has been also discussed in Tsui [29]:

$L_{e}=\int_{-\infty}^{\infty}\left[\frac{(X-T)^{2}}{\frac{U S L-L S L}{2}}\right] d F(X)=\frac{\sigma^{2}+(\mu-T)^{2}}{\frac{U S L-L S L}{2}}$

Pearn, Chang and Wu [30] criticized the indicator of Johnson [28] because it does not take into account the case with asymmetric tolerances and suggested modifying the $L_{e}$ index and introduced a new $L_{e}^{\prime}$ index. Both approaches have been discussed in Abdolshah [31], where is concluded that the loss-based PCIs are more realistic and suitable tools to measure the capability of a process than the traditional ones. However, both approaches have not been extended to the multivariate case.

Eslamipoor and Hosseini-nasab [32] suggested using the signal-to-noise ratio derived from the loss function concept from Taguchi [7] as a practical tool for PCIs. This approach unifies the $C_{p}, C_{p k}, C_{p m}, C_{p k m}$ indices but it is not extended to the multivariate case.

\subsubsection{A dynamic approach}

Up to here, all PCIs are useful to describe the capability of controlled processes but none pays attention to the out-of-control period, where the nonconforming rate is higher than in the in-control period. Thus, Lupo [1] introduced a new univariate PCI, which is related to the proportion of nonconforming parts over a process functioning cycle (during the in-control and out-of control periods) through the cumulative density function.

\subsubsection{PCIs for non-normal measures}

Up to here, it has been supposed that the measured data follows a normal distribution. However, this is not always the case in real production processes. Thus, some PCIs have been also proposed to deal with nonnormal processes. Zwick [33] proposed using PCIs although the product characteristics do not follow a normal distribution by using the $C N_{p x}$ indices. The x means that this method is valid for each univariate PCI $\left(C N_{p}\right.$,

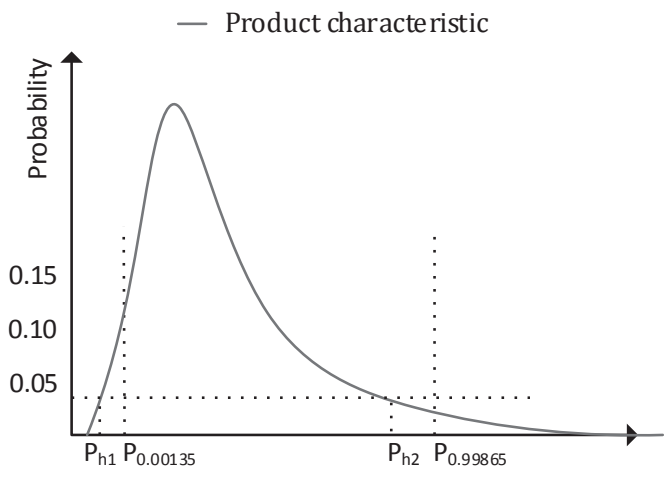

Fig. 7 Definition of the highest density interval in Yang et al. [34].

$\left.C N_{p k}, C N_{p m}, C N_{p m k}\right)$. For example, the $C N_{p}$ can be calculated by following the next formula:

$C N_{p}=\frac{U S L-L S L}{P_{0.99865}-P_{0.00135}}=\frac{S R}{P R}$

where $P_{0.99865}$ and $P_{0.00135}$ are the 99.865 and the 0.135 percentiles of nonconforming data.

Yang et al. [34] showed that the interval defined by 0.135 and 99.865 percentiles may not include the highest probability density interval when dealing with non-symmetric distributions. Yang et al [34] suggest describing the PR for non-normal distributions as the interval $\left[P_{h 1}, P_{h 2}\right]$ that satisfies equation 15 and $f\left(P_{h 1}\right)=$ $f\left(P_{h 2}\right)$, where $f(x)$ is the probability density function. Figure 7 illustrates the intervals defined by Zwick [33] and Yang et al [34] with a non-symmetric probability density function.

$\int_{P_{h 1}}^{P_{h 2}} f(x) d x=0.9973$

Piña-Monarrez, Ortiz-Yañez and Rodríguez-Borbón [35] used the approach explained in Zwick [33] and proposes a methodology to calculate the $C N_{p}, C N_{p k}$ indices when the measures follow Weibull and lognormal distributions.

\section{Multivariate Process Capability Indices}

Multivariate PCIs are statistic indicators used to quantify the goodness of a multivariate process with a single index by relating the variability of the measures of multiple product characteristics with the admissible one. Several multivariate PCIs have been introduced in the literature. 


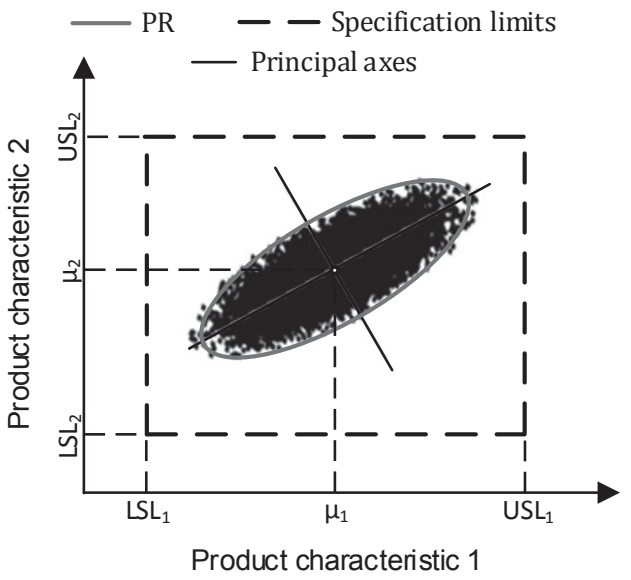

Fig. 8 Representation of the PR main axes.

Henceforth, the PR and the SR are extended to the multivariate case. As in the univariate case, it is accepted that the measures of the product characteristics usually follow a normal distribution. Considering that in the univariate case the $\mathrm{PR}$ is the region that includes $99.73 \%$ of the values drawn from a normal distribution and which is centered on the mean value of the data sample, in the bivariate (and $v$-multivariate) case the $\mathrm{PR}$ is described as the surface ( $v$-dimensional shape) that includes $99.73 \%$ of the values drawn from a binormal (or $v$-multinormal) distribution and which is centered on the mean value of the data sample. This region is mathematical represented by:

$(X-\boldsymbol{\mu})^{\top} \boldsymbol{\Sigma}^{-1}(X-\boldsymbol{\mu})=c^{2}$

where $\boldsymbol{\mu}$ and $\boldsymbol{\Sigma}$ are the mean vector and the variancecovariance matrix, and $c^{2}$ depends on $v$ (e.g. for $v=2$, $\left.c^{2}=11.83\right)$. Usually, it is accepted that $(X-\boldsymbol{\mu})^{\top} \boldsymbol{\Sigma}^{-1}(X-$ $\boldsymbol{\mu})=c^{2}$ (as function of the measured product characteristics) follows a $\chi_{v}^{2}$ distribution with $v$ degrees of freedom. About $99.73 \%$ of the values drawn from a $\chi_{v}^{2}$ distribution with $v$ degrees of freedom are within the region delimited by (16). The $\mathrm{SR}$ is the parallelepiped region whose vertices are delimited by the SLs. Figure 8 shows the distribution of a sample of data for two product characteristics, the $\mathrm{PR}$ and the SR.

Before introducing the multivariate PCIs, it is necessary to understand the influence of the correlation between product characteristics when analyzing the capability of multivariate production processes. In the multivariate case, the correlation between the measured product characteristics plays an important role: The ellipsoidal shape that represents the PR is more -or less- inclined depending on the value of the correlation between the analyzed product characteristics. If there is no correlation between the measures, the axes of the PR are parallel to the Cartesians axes. Figure 8 also shows the main axes of the ellipsoidal PR for a bivariate sample. Thus, only if correlation is taken into account, it is possible to describe properly the PR and, consequently, to evaluate properly the capability of production processes in the multivariate case.

Hereafter, multivariate PCIs from the literature are introduced and classified into two groups. On the one hand, there are multivariate PCIs that do not take into account the correlation between the measured product characteristics and that are obtained through the derivation from univariate PCIs. On the other hand, other multivariate PCIs do take this correlation into account whether through the description of the ellipsoidal shape by its principal component axes, through the comparison between process and specification regions, or through the usage of the cumulative distribution function.

Although in each contribution the authors used their own nomenclature to name the presented PCIs, in this survey all PCIs are named following the $M C_{p x}$ criteria: $M$ means that it is a multivariate PCI and $C_{p x}$ differentiates between $M C_{p}$ (the multivariate PCI only takes into account the size of the PR), $M C_{p k}$ (the multivariate PCI does not only take into account the size of the PR but also its position within the SR) and $M C_{p m}$ (the multivariate PCI also takes into account the position of the target value).

3.1 Multivariate PCIs that do not take into account the correlation between product characteristics.

Derivation from univariate PCIs

In this group we find multivariate PCIs obtained through the derivation from univariate PCIs. Thus, they do not take into account the correlation between the measured product characteristics.

An example is the multivariate PCI presented in Hubele, Montgomery and Chih [36]. It is the arithmetical mean of the univariate PCIs that describe the capability of each single product characteristic. If the quantity of sample data of each product characteristic is the same, the presented PCI is the mean value of all univariate PCIs. This method is valid for each univariate PCI $\left(C_{p}, C P U, C P L, C_{p k}, k, C_{p m}\right.$ and $\left.C_{p m k}\right)$.

Veevers [27] extended the concept of Viability to the multidimensional case by describing the window of opportunity as a volume in order to compare it with 
the volume of the SR:

$V_{r n}=\frac{\text { vol. }(\text { Window of Opportunity })}{\text { vol.(Specification Region })}$

Due to its definition, the window of opportunity can be either positive, null or negative. For this reason, it is necessary to distinguish between the case in which at least one univariate window of opportunity is negative, and the case in which all univariate windows of opportunity are positive.

Plante [37] proposed calculating multivariate PCIs as the geometrical mean of all univariate PCIs that describe the capability of each single product characteristic.

Ch'ng, Quah and Low [38] used the weighted sum of the univariate $C_{p m}$ indices to obtain a multivariate PCI:

$M C_{p m}=\sum_{i=i}^{m} e_{i} C_{p m}$

Siman [39] introduced a new methodology to obtain the capability of multivariate processes by following a directional approach, which is an intuitive method valid for all kind of convex SR (not only valid for rectangular SR). Siman [39] suggested using the unidimensional PCIs $C_{p}, C_{p k}, C_{p m}$ and $C_{p m k}$ in an infinite number of directions with the goal of achieving the most critical one. In practice, it is not possible to work with infinite number of directions. Thus, Siman [39] suggested using equispaced directions (or using parametric programming).

3.2 Multivariate PCIs that do take into account the correlation between product characteristics

In the literature there are several multivariate PCIs that do consider the influence of the correlation between the measured product characteristics. In order to classify this kind of multivariate PCIs, three groups are proposed:

- Multivariate PCIs based on principal component analysis.

- Multivariate PCIs based on the relation between PR and SR.

- Multivariate PCIs based on the inverse function of the cumulative distribution function.

\subsubsection{Multivariate PCIs based on principal component analysis}

Wang and Chen [40] proposed using the principal component analysis (PCA) built on Tongs theorem [41]

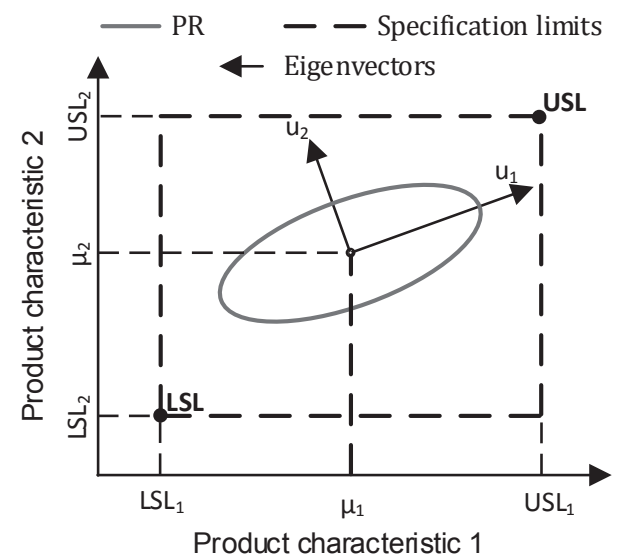

Fig. 9 PR described with its eigenvectors.

in order to obtain multivariate PCIs. Thanks to the PCA method it is possible not only to obtain the main axes (eigenvectors) of the PR (ellipses, ellipsoids, ellipsoidal shapes) but also to obtain a diagonal variancecovariance matrix that describes the PR by eliminating the correlation between the measured product characteristics. Therefore, the modified product characteristics derived with PCA are uncorrelated.

Figure 9 shows the eigenvectors $\left(\mathbf{u}_{\mathbf{i}}\right)$ that describe the main axes of the ellipse in a two-dimensional case. Both axes are orthogonal and uncorrelated.

Thus, the multivariate PCIs presented in Wang and Chen [40] $\left(M C_{p}, M C_{p k}, M C_{p m}\right.$ and $\left.M C_{p m k}\right)$ can be calculated by obtaining the axes of the PR and by calculating the univariate PCIs $\left(C_{p}, C_{p k}, C_{p m}\right.$ and $\left.C_{p m k}\right)$ in the direction of each axis. Afterwards, the multivariate PCIs can be obtained by calculating the geometrical mean of all the univariate and uncorrelated PCIs.

In order to calculate the PCIs corresponding to each principal componnet, Wang and Chen [40] proposed transforming the SR and the target values by using equations 19 to 21 .

$$
\begin{aligned}
& \mathbf{L S L}_{\mathbf{P C}}=\mathrm{U} \cdot \mathbf{L S L} \\
& \mathrm{USL}_{\mathbf{P C}}=\mathrm{U} \cdot \mathrm{USL} \\
& \mathbf{T}_{\mathbf{P C}}=\mathbf{U} \cdot \mathbf{T}
\end{aligned}
$$

where $\mathbf{L S L}_{\mathbf{P C}}=\left(L S L_{P C_{1}}, \ldots, L S L_{P C_{v}}\right), \mathbf{U S L}_{\mathbf{P C}}=$ $\left(U S L_{P C_{1}}, \ldots, U S L_{P C_{v}}\right)$ and $\mathbf{T}_{\mathbf{P C}}=\left(T_{P C_{1}}, \ldots, T_{P C_{v}}\right)$ are, respectively, the vectors of the lower and upper specification limits and the target values corresponding to each principal component. $\mathbf{L S L}=\left(L S L_{1}, \ldots, L S L_{v}\right)$ and USL $=\left(U S L_{1}, \ldots, U S L_{v}\right)$ and $\mathbf{T}=\left(T_{1}, \ldots, T_{v}\right)$ 
are, respectively, the vectors of the LSL, USL and target values corresponding to each product characteristic. $\mathbf{U}$ is the rotation matrix with the eigenvectors of the variance-covariance matrix.

It is important to notice that the rotation of the PR also involves a rotation of the SR. However, the method proposed in Wang and Chen [40] only rotates the SR vertices (LSR and USR) by using the midpoint of the $\mathrm{PR}$ as the fix rotation point. Thus, the adapted SR remains parallel to the Cartesians axes. Although the elimination of the correlation between product characteristics, this method generates problems related to the rotation of the SR because of the modification of its size [42].

Wang and Du [43] improved the approach in Wang and Chen [40] by reducing the number of necessary eigenvectors that must be taken into account to describe the capability of a multivariate process. Wang and Du [43] suggested considering the ratio of each eigenvalue to the summation of the eigenvalues -which describes the proportion of variability associated with each principal component variable- and selecting only the eigenvectors that contribute to most of the process variability. The eigenvector reduction is possible thanks to the Jacksons theorem [44] that checks if each eigenvector must be taken into account.

Wang [45] proposed weighting the uncorrelated PCIs by considering its relative importance through their eigenvalues $\left(\lambda_{i}\right)$ :

$M C_{p x}=\left(\prod_{i=1}^{v} C_{p x ; P C i}\right)^{\frac{\lambda_{i}}{\sum_{j=1}^{v} \lambda_{j}}}$

Shinde and Khadse [42] showed that the modified SR proposed in Wang and Chen [40] was not correct. As it has been stated, Wang and Chen [40] proposed a modification of the SLs but the SR remained parallel to the Cartesians axes. However, a correct rotation of the SR must generate an inclined SR. Figure 10 shows the correct rotation of the SR proposed in Shinde and Khadse [42] and the SR proposed in Wang and Chen [40] .

In order to solve the problem related to the rotation of the SR, Shinde and Khadse [42] introduced two new multivariate PCIs: the $M_{p_{1}}$ and the $M_{p_{2}}$ indices (analog to the $M C_{p}$ and $M C_{p k}$ indices). If the $M_{p_{1}}$ index is bigger than (or equal to) 0.9973 , the process is potential capable. If $M_{p_{2}}$ is bigger than (or equal to) 0.9973 , the process is actually capable. In order to calculate these indices, Shinde and Khadse [42] proposed an empirical approach using two Monte Carlo generations of data.

González and Sánchez [26] proposed another multivariate PCI obtained by using the PCA method. With this approach, it is possible to calculate a multivariate

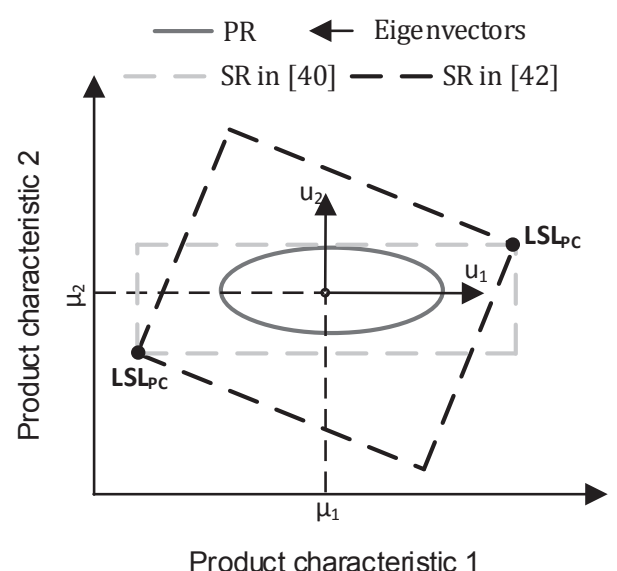

Fig. 10 Rotated PR and SR.

PCI that represents how much can increase the standard deviation before the process is not capable by considering the maximal standard deviation of the diagonal variance-covariance matrix. It is interesting to point out, that the logic used in this multivariate PCI is the same as the used in the univariate $C_{n}$ index.

In this regard, Perakis and Xekalaki [46] introduced a multivariate PCI which was also valid for unilateral specification limits.

Tano and Vännman [47] introduced a multivariate PCI that uses not only the PCA method (with the correct inclination of the $\mathrm{SR}$ ) but also normalizes the original sample by modifying and adapting it to the interval $[-1,1]$. The diagonal variance-covariance matrix of the normalized sample is calculated in order to obtain its eigenvalues and eigenvectors. In order to obtain this multivariate PCI, Tano and Vännman [47] affirmed that only the biggest eigenvalue $\left(\sigma_{P C A_{1}}\right)$ is necessary because in this approach only the width of the PR is taken into account.

Last but not least, Dharmasena and Zeephongsekul [48] introduced a multivariate PCI based on the PCA method by generalizing some existing multivariate indices based on the PCA method proposed by several authors.

\subsubsection{Multivariate PCIs based on the relation between process and specification regions}

The PR and SR can be represented with ellipsoidal and parallelepiped shapes, respectively. The proposed multivariate PCIs in this section compare the size of these shapes considering their original or modified representations presented in different articles. 


\subsubsection{Relation between original $\mathrm{PR}$ and original $\mathrm{SR}$}

Chen [49] presented a multivariate PCI by comparing the sizes of the original PR and SR. This multivariate $\mathrm{PCI}$ only gives information of the $\mathrm{PR}$ size in comparison with the size of the SR but it does not consider the position of the PR within the SR.

Das and Dwivedi [50] introduced a multivariate PCI valid for non-normal and correlated product characteristics assuming multivariate $g$-and- $h$ distributions. However, the problem using $g$-and- $h$ distributions is the high computation that is required.

Ciupke [51] introduced a multivariate PCI that compares the size of the original PR and SR and which allows to analyze the capability of both normal and nonnormal product characteristics. Taking into account that the PR of non-normal multivariate product characteristics cannot be described by (16), Ciupke [51] suggested using one-side models to determine the PR shape.

\subsubsection{Relation between original $\mathrm{PR}$ and modified SR}

In this group, we find all methods that compare the size of the original PR and a modified SR using (23). If the sample data follows a normal distribution, the original PR is described by (16).

$M C_{p x}=\frac{\text { vol. }(\text { modified } S R)}{\text { vol. }(\text { original } P R)}$

Chan, Cheng and Spiring [52] proposed using an ellipsoidal shape to represent the SR. Taam, Subbaiah and Liddy [53] improved the approach presented in Chan, Cheng and Spiring [52] and described the modified SR as the biggest ellipsoid (parallel to the Cartesians axes) that can be fitted into the original SR and which is centered at the target value (see figure 11). We can see that the modified SR is tangential to the original rectangular one.

Braun [54] took into account the effect of the correlation between product characteristics while modifying the SR. In this contribution, the modified SR is an ellipsoidal shape centered on the midpoint of the original $\mathrm{SR}$, tangential to the rectangular $\mathrm{SR}$ and parallel to the main axes of the PR. Braun [54] proposed a PCI considering both, the size of the $\mathrm{PR}$ and its position within the SR. Thus, a correlation coefficient, which depends not only on the position of the PR but also on the middle point of the SR was also presented. This approach is focused in the case in which the target value is the midpoint of the SR. Shaoxi et al. [55] improved the approach presented in Braun [54] by proposing a new correlation coefficient.

Pan and Lee [56] also suggested considering the existing correlation between the measures of the product characteristics in order to obtain a $M C_{p m}$ index. Here,

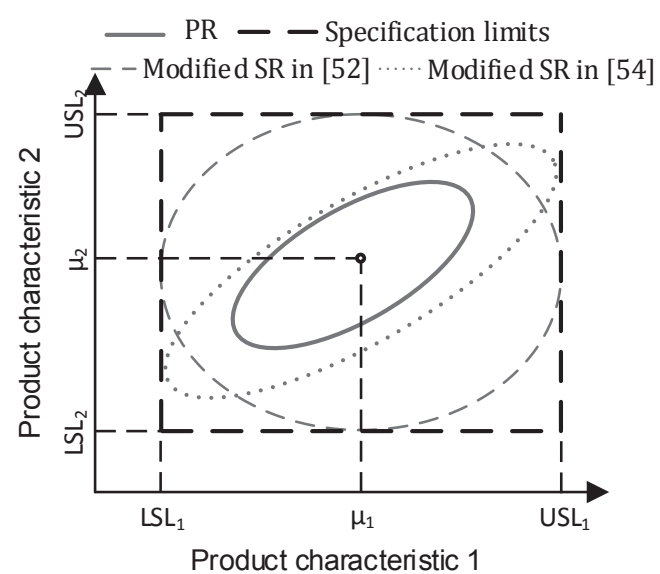

Fig. 11 SR and modified SR as described in Chan, Cheng and Spiring [52]. and in Braun [54]

a modified SR, which is also inclined but it is now centered in the target value (which cannot be the center of the SR) is proposed. The approach in Pan and Lee [56] is extended to the non-normal case in Pan, Li and Shih [57] by using a weighted standard deviation method to approximate the original probability density function with segments from $2^{v}$ multivariate normal distributions.

Jalili, Bashiri and Amiri [58] introduced another multivariate PCI, which is also valid for unilateral specification processes. In Jalili, Bashiri and Amiri [58] the $\mathrm{PR}$ is divided into two different parts: the part that is within the specification region $(\mathrm{CV})$ and the part that is without the specification region ( $\mathrm{NCV})$. Then, the relation between $\mathrm{CV}$ and $\mathrm{NCV}$ is used to obtain a multivariate PCI. With this method, not only the volume of the PR but also its position within the $\mathrm{SR}$ is taken into account. However, the relation between the proportion of nonconforming parts (NCP) and the PCI is not the one obtained per definition through the cumulative distribution function (see Castagliola [59]).

\subsubsection{Relation between modified $\mathrm{PR}$ and original SR}

Shahriari, Hubele and Lawrence [60] introduced a multivariate process capability vector that can be calculated with (24) by comparing the original SR with a modified PR.

$M C_{p x}=\frac{\text { vol. }(\text { original } S R)}{\text { vol. }(\text { modified } P R)}$

The modified PR proposed in Shahriari, Hubele and Lawrence [60] is a parallelepiped shape which is tangen- 


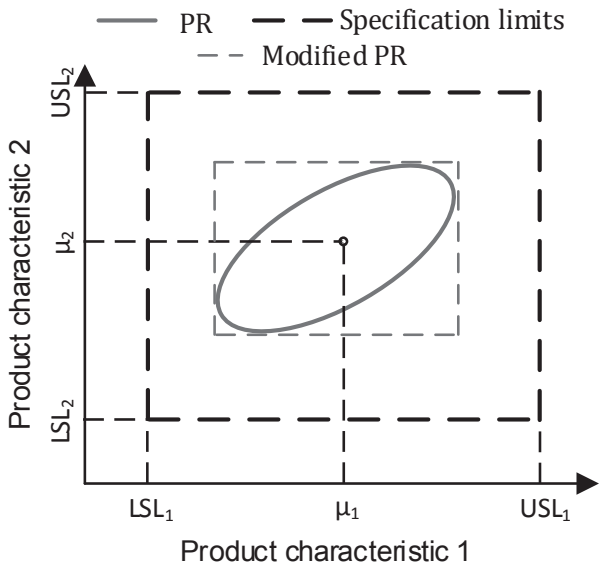

Fig. $12 \mathrm{PR}$ and modified $\mathrm{PR}$ as described in Shahriari, Hubele and Lawrence [60].

tial to the original PR and parallel to the Cartesians axes (see figure 12).

The process capability vector has three components: The first component $\left(C_{p M}\right)$ gives information of the relation between the sizes of the original $\mathrm{SR}$ and the modified PR; the second component $(\mathrm{PV})$ is a $p$-value computed to test the null hypothesis $\mu=\mu_{0}$ that gives information of the relative location of the $\mathrm{PR}$ within the $\mathrm{SR}$; the third component gives additional information about the location of the modified PR within the SR.

Niavarani, Noorossana and Abbasi [61] developed the approach from Shahriari, Hubele and Lawrence [60] and suggested using a modified PR parallel to the ellipsoid axes to calculate the first component of the capability vector.

3.2.2.4 Relation between modified PR and modified $\mathrm{SR}$

Grau [62] proposed a new method to calculate the capability of multivariate processes. This method considers the possibility that the target value and the midpoint of the PR are not the same point. This contribution introduces four different shapes to represent the modified SR and PR in order to calculate the following

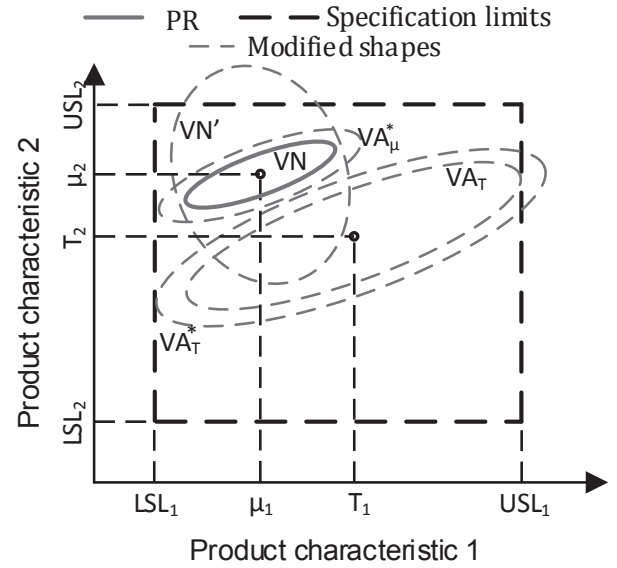

Fig. 13 Modified shapes in Grau [62].

multivariate PCIs:

$$
\begin{aligned}
& M C_{p}=\left(\frac{V A_{T}}{V N}\right)^{\frac{1}{v}} \\
& M C_{p k}=\left(\frac{V A_{\mu} *}{V A_{T^{*}}}\right)^{\frac{1}{v}} M C_{p} \\
& M C_{p m}=\left(\frac{V A_{T}}{V N^{\prime}}\right)^{\frac{1}{v}} \\
& M C_{p m k}=\left(\frac{V A_{\mu} *}{V A_{T^{*}}}\right)^{\frac{1}{v}} M C_{p m}
\end{aligned}
$$

$V N$ is the original PR. $V A_{T}$ is the maximal homothetic ellipsoidal shape which is centered on the target value and that fits (tangential) within the original SR. $V A_{\mu} *$ is the maximal homothetic ellipsoidal shape which is centered on the PR mean and that fits (tangential) within the original SR. $V A_{T} *$ is the maximal homothetic ellipsoidal shape which is centered on the target and that is tangential to the same specification limit as $V A_{\mu} * . V N$ is the ellipsoidal shape that is centered on the process output characteristic mean and whose variance-covariance matrix can be calculated with (29) where $Q(\mu)$ is a mathematical function that also takes into account the position of the target value. All this shapes are represented in figure 13.

$$
\boldsymbol{\Sigma}_{Q}=\boldsymbol{\Sigma}+Q(\boldsymbol{\mu}) Q(\boldsymbol{\mu})^{\top}
$$

\subsubsection{Multivariate PCIs based on the inverse function of the cumulative distribution function}

All multivariate PCIs included in this group use the alternative definition for the $C_{p k}$ index proposed in 
Castagliola [59]. This definition takes into account the relation between the proportion of nonconforming parts (NCP) of the analyzed process and the value of the $C_{p k}$ index. The proportion of nonconforming parts under the $L S L\left(p_{L S L}\right)$ can be calculated using the CPL index (3) and the cumulative distribution function $(\Phi)$ of the standardized normal distribution $N(0,1)$ :

$p_{L S L}=\Phi\left(\frac{L S L-\mu}{\sigma}\right)=\Phi(-3 C P L)$

Inverting the cumulative distribution function, the relation between the $C P L$ index and $p_{L S L}$ can be obtained:

$C P L=-\frac{1}{3} \Phi^{-1}\left(p_{L S L}\right)$

This logic can also be used by considering the proportion of nonconforming parts above the $U S L\left(p_{L S L}\right)$. Then, the relation between the $C_{p k}$ index and the proportion of nonconforming parts can be described by:

$C_{p k}=\frac{1}{3} \min \left\{-\Phi^{-1}\left(p_{L S L}\right),-\Phi^{-1}\left(p_{U S L}\right\}\right.$

Bothe [23] introduced a $M C_{p k}$ index based on this premise. In order to calculate it, first it is necessary to obtain for each product characteristic, namely $i$, the probability that a measure of the product characteristic is within the specification limits $\left(p_{i}\right)$. After it, it is necessary to obtain the total proportion $\left(p_{\text {total }}\right)$ of conforming parts with:

$p_{\text {total }}=\prod_{i=1}^{n} p_{i}$

Then, the total proportion of nonconforming parts $\left(p_{\text {total }, N C P}\right)$ can be obtained with:

$p_{\text {total }, N C P}=1-p_{\text {total }}$

With the inverse cumulative normal distribution function, $\Phi$, it is possible to transform the total proportion of nonconforming parts into a multivariate PCI.

If many product characteristics are considered simultaneosuly, the total proportion of conforming parts $\left(p_{\text {total }}\right)$ tends to be null. In order to avoid it, Bothe [23] also presented a normalized version of this approach. However, this approach is valid only if all output characteristics are uncorrelated.

Pearn et al. [63] suggested calculating the total proportion of nonconforming parts with (35) and then, transforming it to a $M C_{p k}$ index by using the relation between the proportion of nonconforming parts and the $C_{p k}$ index in Castagliola [59].

$p_{\text {total }, N C P}=\prod_{i=1}^{v}\left(1-C P_{i}\right)$

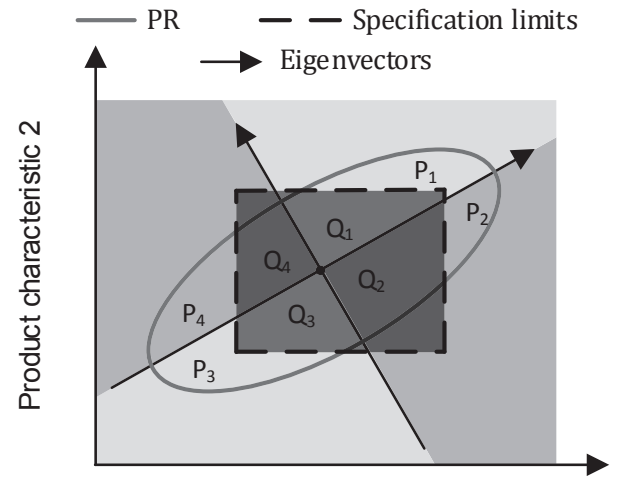

Product characteristic 1

Fig. 14 Process region sub-areas as stated in Castagliola and Garcia Castellanos [64].

The good point of the approaches in Bothe [23] and in Pearn et al. [63] is that given a $M C_{p k}$ value, the expected proportion of nonconforming parts can be estimated. Nevertheless, both approaches do not take into account the correlation between the product characteristics, and thus, the $p_{\text {total.NCP }}$ is not properly estimated. In order to transform the approach proposed in Bothe [23] and in Pearn et al. [63] into a valid method to analyze correlated product characteristics, the $\mathrm{M} C_{p k}$ index for the bivariate $\left(B C_{p k}\right)$ and correlated case was presented in Castagliola and Garcia Castellanos [64]. Castagliola and Garcia Castellanos [64] followed the nomenclature of the PCA method in order to obtain the main axes of the ellipse that represents the PR. These two axes divide the PR in four areas $\left(A_{1}, A_{2}, A_{3}, A_{4}\right)$. The probability that the measures of the product characteristics are within one of these areas is the same for each one $\left(P\left(X \in A_{i}\right)=1 / 4\right)$.

The $A_{i}$ areas are also divided in two differentiated sub-areas (see figure 14). On the one hand, $Q_{1}, Q_{2}$, $Q_{3}$ and $Q_{4}$ are the sub-areas that are within the SR. On the other hand, $P_{1}, P_{2}, P_{3}$, and $P_{4}$ are the subareas that are outside the SR. The probabilities that the measures of the product characteristics are within one of this areas $\left(Q_{i}\right.$ and $\left.P_{i}\right)$ are called $q_{i}$ and $p_{i}$ :

$p_{i}=\frac{1}{4}-q_{i}$

The total proportion of nonconforming parts is the sum of all single $p_{i}$ probabilities $\left(p_{\text {total }, N C P}=p_{1}+p_{2}+\right.$ $\left.p_{3}+p_{4}\right)$. 
Then, the multivariate PCI can be calculated with the inverse cumulative normal distribution function:

$$
\begin{aligned}
B C_{p k}=\frac{1}{3} \min \{ & -\Phi^{-1}\left(2 p_{1}\right),-\Phi^{-1}\left(2 p_{2}\right), \\
& \left.-\Phi^{-1}\left(2 p_{3}\right),-\Phi^{-1}\left(2 p_{4}\right)\right\}
\end{aligned}
$$

If the four probabilities $\left(p_{1}, p_{2}, p_{3}\right.$ and $\left.p_{4}\right)$ are equal, the total proportion of nonconforming parts is minimal. In this case the $B C_{p k}$ is maximal.

The method presented in Castagliola and Garcia Castellanos [64] is extended to the $v$-variate case in Shiau et al. [65]. Here, the PR ellipses have $v$ axes and are divided into $2^{v}$ Euclidian shapes. The proposed $M C_{p k}$ index of Shiau et al. [65] is:

$$
\begin{gathered}
M C_{p k}=\frac{1}{3} \min \left\{-\Phi^{-1}\left(2^{v-1} p_{1}\right),-\Phi^{-1}\left(2^{v-1} p_{2}\right),\right. \\
\left.\ldots,-\Phi^{-1}\left(2^{v-1} p_{2^{k}}\right)\right\}
\end{gathered}
$$

In order to obtain $p_{1}, p_{2}, \ldots, p_{2^{k}}$, Shiau et al. [65] suggested using Montecarlo replications. Furthermore, in Shiau et al. [65] the approach presented in Castagliola and Garcia Castellanos [64] was criticized because it is not scale invariant. Thus, Shiau et al. [65] proposed scaling the sample data and the specification limits. With this modification it is possible to calculate not only a $M C_{p k}$ but also a $M C_{p}$ index.

Abbasi and Niaki [66] also suggested using the inverse cumulative normal distribution function but to describe the capability of non-normal multivariate production processes. First, it is necessary to transform the measures by using a root transformation technique. Then, a Monte Carlo simulation method has to be used to estimate the proportion of nonconforming parts of the process. However, the approach suggested in $\mathrm{Ab}-$ basi and Niaki [66] does not consider the smaller-the better or unilateral case.

Gu et al. [67] suggested that a capability index for evaluating the performance of multivariate processes must be yield-based. In other words, a PCI must have a clear relationship with the process yield. Indices, such as the $C_{p k}$, describe the process yield by considering only the nonconforming parts of a process in one direction. However, it does not represent the real process yield. Taking it into account, Gu et al. [67] introduced two new PCIs: the $E C_{p k}$ for univariate processes and the $M E C_{p k}$ for multivariate processes. Both indices use the original definition of the $C_{p k}$ index and adapt it by obtaining the process yield through the cumulative distribution function. This approach requires some accurate high-precision calculation techniques to compute the multivariate cumulative distribution function.

Last, de-Felipe et al. [68] also introduced a multivariate PCI $\left(M C_{p k}\right)$ based on the relation between the proportion of nonconforming parts and the $C_{p k}$ in
Castagliola [59] but suggested using the multivariate normal cumulative distribution function to calculate the total proportion of nonconforming parts.

\section{Clustering and discussing univariate and multivariate PCIs}

In this section all PCIs introduced in this article are clustered in order to obtain an overview. To cluster all the explained PCIs, table 1 is proposed. It may help the reader to summarize the important characteristics of each PCI. The rows show all contributions, which are in chronological order. In the columns we can distinguish three classifications:

The first classification gives information of the type of process data that can be analyzed with each PCI. It is important to differentiate not only between univariate and multivariate processes but also between processes with normal and non-normal distributed product characteristics.

The second classification gives information of the method that the authors used in order to calculate the PCIs. On the one hand, there are univariate and multivariate PCIs that use the traditional definition of univariate $\mathrm{PCIs}$, namely without taking into account the correlation between the product characteristics. On the other hand, there are multivariate PCIs that do consider the correlation between the measures of the product characteristics and that are obtained by three different ways: First, there are multivariate PCIs based on the PCA method. Second, there are multivariate PCIs based on the comparison between modified and original SR and PR. Third, there are multivariate PCIs based on the transformation of the proportion of nonconforming parts of the analyzed process into a PCI with help of the inverse function of the cumulative distribution function.

The third classification shows the information that the PCIs give. First, there are PCIs that give information about the width of the PR in comparison with the width of the SR. Second, there are PCIs that also consider the position of the PR within the SR. Third, there are PCIs that also consider the position of the target value. Finally, there are PCIs that also prognosticate the proportion of nonconforming parts of the process.

\subsection{Discussion: univariate PCIs}

In this section, we are going to discuss critically the univariate PCIs that have been introduced in this article. Univariate PCIs were introduced in the literature to analyze the capability of production processes with 
Table 1 Overview of univariate and multivariate PCIs

Kane (1986) [19]

Chan, Cheng and Spiring (1988) [24]

Chan, Cheng and Spiring (1991) [52]

Hubele, Montgomery and Chih (1991) [36] Johnson (1992) [28]

Pearn, Kotz and Johnson (1992) [25]

Taam, Subbaiah and Liddy (1993) [53] Chen (1994) [49]

Zwick (1995) [33]

Shahriari, Hubele and Lawrence (1995) [60 Veevers (1998) [27]

Wang and Chen (1998) [40] Bothe (1999) [23]

Wang and Du (2000) [43] Braun (2001) [54]

Castagliola and Garcia Castellanos (2005) [64] Wang (2005) [45]

Pearn, Chang and Wu (2006) [30] Grau (2007) [62]

Shinde and Khadse (2009) [42]

González and Sánchez (2009) [26 Pan and Lee (2010) [56

Abbasi and Niaki [66]

Perakis and Xekalaki (2011) [46]

Pearn et al. (2011) [63]

Jalili, Bashiri and Amiri (2012) [58]

Niavarani, Noorossana and Abbasi (2012) [61]

Shiau et al. (2013) [65]

Shaoxi et al. (2013) [55]

Das and Dwivedi (2013) [50]

Tano and Vännman (2013) [47] Siman (2014) [39]

Dharmasena and Zeephongsekul (2015) [48] Ciupke (2015) [51]

Yang et al. (2015) [34] Lupo (2015) [1]

Gu et al. (2015) [67]

Piña-Monarrez, Ortiz-Yañez and Rodríguez-Borbón(2015) [35]

Eslamipoor and Hosseini-nasab (2016) [32]

Pan, Li and Shih (2016) [57]

de-Felipe et al. (2016) [68]

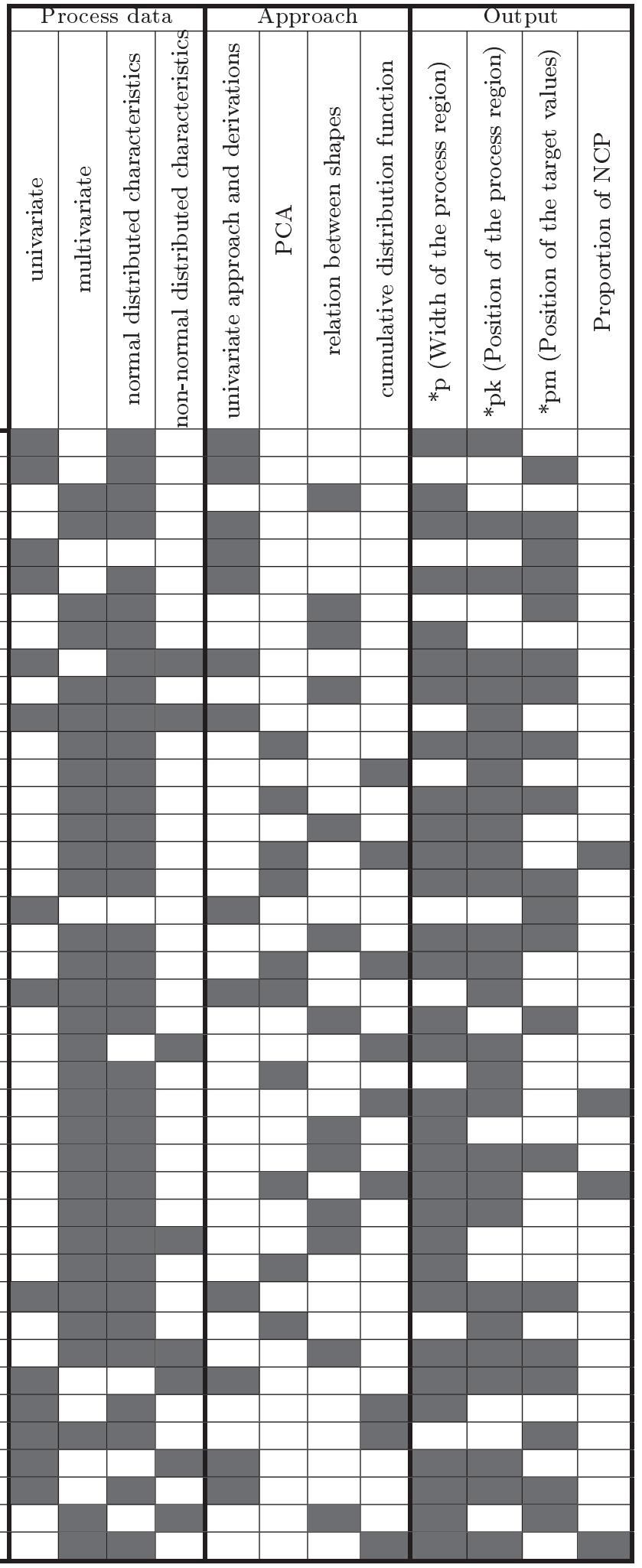



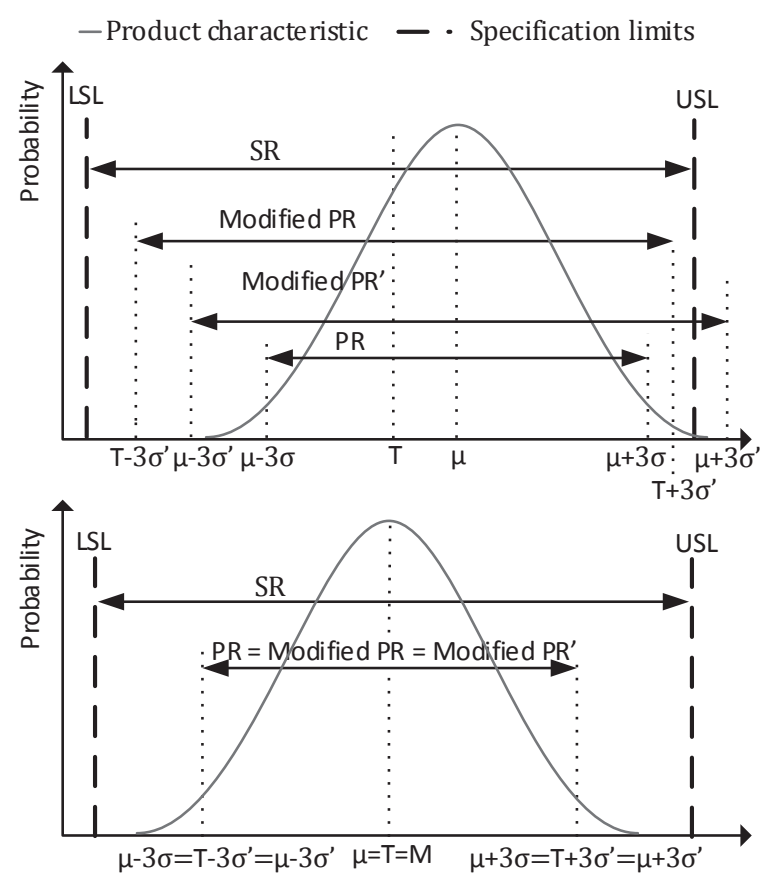

Fig. 15 Discussion of univariate PCIs using two cases of study.

only one product characteristic. From all the existing PCIs, the $C_{p k}$ index is widely used in the industry. For this reason, particular attention has been paid to this univariate PCI.

Hereafter, we use two different cases of study (see figure 15) to discuss critically some univariate PCIs presented in this article. In the first case, the measures of the product characteristic follow a normal distribution which is not centered on the target value $(\mu=0.21875$, $\sigma=0.2083)$ In the second case, the measures of the product characteristic follow a normal distribution centered on the target value, which is also the midpoint of the $\operatorname{SR}(\mu=0, \sigma=0.2083)$. For both cases of study $L S L=-1, U S L=1$ and $T=0$.

Table 2 summarizes for both cases the following univariate PCIs: $C_{p}, C P U, C P L, k, C_{p k}, C_{p m}, C_{p m k}, V_{p}$, $C_{n}$. It is possible to see that depending on the PCI used we can obtain different values of the capability of the process.

In the first case, the original PR, the modified PR and PR' have different widths because of the non-centered position of the PR within the SR. Thus, the values of the $C_{p}, C_{p k}, C_{p m}, C_{p m k}$ indices (see table 2) are different. In the second case, the original PR and the modified PR and PR' have the same width and are placed on
Table 2 Comparison of univariate PCIs

\begin{tabular}{lcc}
\hline & Case 1 & Case 2 \\
\hline$C_{p}$ & 1.60 & 1.60 \\
$C P U$ & 1.25 & 1.60 \\
$C P L$ & 1.95 & 1.60 \\
$k$ & 0.22 & 0.00 \\
$C_{p k}$ & 1.25 & 1.60 \\
$C_{p m}$ & 1.10 & 1.60 \\
$C_{p m k}$ & 0.86 & 1.60 \\
$V_{r}$ & 0.38 & 0.38 \\
$C_{n}$ & 1.60 & 1.60 \\
\hline
\end{tabular}

the same position. Thus, there is no difference between these univariate PCIs.

Thanks to this example, it is possible to understand the benefits of using PCIs that take into account the position of the $\mathrm{PR}$ within the $\mathrm{SR}$. If the $\mathrm{PR}$ is not centered in relation to the $\mathrm{SR}$, the capability of the process is not properly described with indices that do not take into account the mean value of the measures. For example, if we look at table 2, we can see that in both cases the $C_{p}$ value is the same although the PR is not placed in the same place. Nevertheless, the $C_{p k}$ in the first case suggests that the PR is not centered within the SR. As we can also see, the $V_{r}$ and the $C_{n}$ indices do not describe the capability of off-centered processes (in both cases the value of both indices is the same). Thus, taking only into account the $C_{p}, V_{r}$ or $C_{n}$ indices, a process with product characteristics outside the SR can be described as capable although it does not comply with the capability requirements. In this example it is also possible to see that depending on the PCI used to describe the capability of a process (see for example the $C_{p k}$ and the $C_{p m k}$ indices in case 1) a process can be described as capable or non-capable.

\subsection{Discussion: multivariate PCIs}

In this section we discuss critically the multivariate PCIs introduced in this article in order to identify conceptual and methodological problems of each group (see table 1) and to identify multivariate PCIs useful for the industry. In our discussion we are going to focus on the following criteria: calculation method; globality of the index; relation to proportion of nonconforming parts; and robustness of the index.

The calculation method is defined as the way used to calculate the multivariate PCI. As we have seen, it is possible to distinguish between four different methods: derivation from univariate PCIs, elimination of the cor- 
relation through the usage of the PCA method, comparison between shapes, and usage of the cumulative distribution function. We accept that obtaining multivariate PCIs through the derivation from univariate PCIs is an easy way to calculate multivariate PCIs. Nevertheless, the other three methodologies are not so straightforward to calculate because they are based on high complex mathematical functions.

The globality of the index is defined as the ability to synthesize the capability of multiple product characteristics with a single index. We have seen that multivariate PCIs are on focus of research field and lots of approaches and methods are presented recently in the literature. It seems to be a huge interest on evaluating production processes with multiple product characteristics in a global way by summarizing the capability relating several product characteristics with a single index. Thus, all the contributions related to multivariate PCIs discussed in this survey are dealing with this topic.

The third criterion is the relation between the value of the multivariate PCI and the proportion of nonconforming parts of the analyzed production process. Establishing relationship between process capability indices and proportion of nonconformance has been studied extensively in the literature [69]. From our point of view, trying to find a methodology to represent the capability of multivariate processes in a global way, sometimes the original objective of the PCIs is being dismissed: PCIs were introduced in the literature to describe the capability of a process; or in other words, to estimate the ability of a process to produce outputs within the SL. Thus, if univariate PCIs, such as the $C_{p k}$ index, are indirectly indicators of the proportion of conforming and nonconforming parts of production processes, multivariate PCIs must deal with the same goal. Taking a look to table 1 , it is possible to see that the authors that are thinking in terms of proportion of nonconforming parts are those who are proposing approaches based on the inverse function of the cumulative distribution function.

By robustness of the index is meant the consistence of the value of the multivariate PCI when analyzing a process. Some approaches, such as the ones sampling using Monte Carlo, present inconsistency of the values because different values are obtained depending on the Monte Carlo simulation.

Hereafter we discuss the multivariate PCIs presented in this article taking into account these four criteria.

First, we focus on the multivariate PCIs obtained through the derivation from univariate PCIs. Thanks to this methodology, multivariate PCIs are obtained directly from the univariate ones through easy computation methods such as geometrical and arithmeti- cal means. Thus, these indices present robust behaviors and describe the capability of the process in a global way with a single index. Nevertheless, there is no relation between these indices and the proportion of nonconforming parts of the analyzed process. Imagine a multivariate production process with some product characteristics that present good capability behaviors and other product characteristics that present poor capability behaviors. Using the mean value of the univariate $\mathrm{PCIs}$ to calculate a multivariate $\mathrm{PCI}$, this index would be an intermediate value that may suggest that the capability of the process is acceptable, although some product characteristics are not capable. If we accept that processes that have non-capable product characteristics must be described as non-capable processes, using this methodology may lead to misunderstandings.

Second, we focus now on the methods based on the relation between PRs and SRs. Authors that suggest comparing the sizes of these regions, are just testing if the PR can be fitted within the SR but the multivariate $\mathrm{PCI}$ s that they are suggesting do not give information about the position of the PR within the SR, and thus, about the proportion of nonconforming parts of the process. For example, we can take the proposed index in Chen [49], which suggests comparing the sizes of the original PR and SR. Imagine that the variability of the measured data is really small, and thus we have a really small PR in comparison with the SR. In this case, when comparing the sizes of the PR and the SR we will obtain a rate higher than one and thus, we will believe that the process is capable. But, actually, with this approach the position of the PR within the SR has not been taken into account. Following with this example, if the small PR is centered on the middle of the SR the proportion of nonconforming parts is going to be really small; but if the PR is off-centered, the proportion of nonconforming parts is going to be higher. The multivariate PCI in Chen [49] does not distinguish between these two situations (one with a low rate of nonconforming parts and another one with a huge rate of nonconforming parts), and thus, it is not an effective indicator of the proportion of conforming and nonconforming parts of a production process. In order to deal with this problem, several authors suggested modifying either the PR or the SR. With these modifications, they are trying to solve this problem but they are forgetting the original objective of the PCIs: to describe the proportion of conforming and nonconforming parts of the process. By modifying these regions, the information obtained with the ratio between modified PR and SR does not describe the real proportion of nonconforming parts of the process analyzed. 
Third, we focus now on the multivariate PCIs based on the PCA method. As we have seen, authors that suggest using the PCA method to transform the original variance-covariance matrices, which describe the original PR, into a diagonal and uncorrelated- matrix are dealing with eliminating the correlation between the measured product characteristics. With this approach, the whole problem is moved to a new system of coordinate axes defined by the eigenvectors [70]. Once the product characteristics are uncorrelated, these authors suggest combining univariate PCIs to calculate the capability of each direction described by each eigenvector. Using this method, each direction is studied individually; and, afterwards, the capability of each direction is weighted to obtain the global capability of the process. Thus, with this method, a global ratio (comparison of uncorrelated PR and SR) is obtained. However, this ratio is not related to the proportion of nonconforming parts of the process; as with the PCIs of the first group (derivation from univariate PCIs). Moreover, while calculating PCIs in the directions of the eigenvectors, these authors encounter difficulties because they are forced to rotate the SR and the rotation of the SR is not straightforward to calculate. Furthermore, the rotation of the PR leads to modifications of the SR and this leads to the same problem as in the methods of the second group (relation between PRs and SRs): by modifying these regions, the information obtained with the ratio between PR and SR does not describe the real proportion of nonconforming parts of the analyzed process.

Finaly, we discuss now the multivariate PCIs based on the inverse function of the cumulative distribution function. Using the alternative definition for the $C_{p k}$ index proposed in Castagliola [59] is possible to relate the proportion of nonconforming parts of a process with a multivariate PCI. The difficulties in this methodology are related to the way to calculate the proportion of nonconforming parts of the process. While Bothe [23] and Pearn et al. [63] introduced this method to evaluate the capability of multivariate production processes with uncorrelated product characteristics, Shiau et al. [65] and de-Felipe et al. [68] dealt with the case in which the $v$ product characteristics are correlated. On the one hand, Shiau et al. [65] calculated the proportion of nonconforming parts of a process by replicating the measured sample using Monte Carlo. This methodology deals with inconsistence of the value of the multivariate PCI because different values are obtained depending on the Monte Carlo simulation used. The approach in deFelipe et al. [68] solved this problem by calculating the proportion of nonconforming parts using the multivariate normal cumulative distribution function. Moreover, it is also important to point out that while the method
Table 3 Comparison of multivariate PCIs

\begin{tabular}{|c|c|c|c|c|}
\hline & 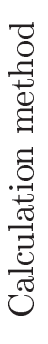 & 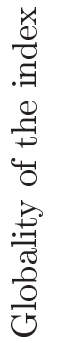 & 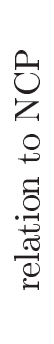 & 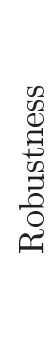 \\
\hline Derivation from univariate PCIs & + & + & - & + \\
\hline $\mathrm{PCA}$ & & + & - & + \\
\hline Relation between shapes & - & + & - & + \\
\hline Cumulative distribution function & - & + & + & $+^{1}$ \\
\hline
\end{tabular}

${ }^{1}$ Not all multivariate PCIs in this group are robust

proposed in Shiau et al. [65] describes the proportion of nonconforming parts in the critical $2^{v}$-Euclidian shapes described by the eigenvectors of the $\mathrm{PR}$, the $M C_{p k}$ in de-Felipe et al. [68] describes the proportion of nonconforming parts above and under the SL. Thus, the $M C_{p k}$ in de-Felipe et al. [68] uses the same logic (relation between PCI and proportion of nonconforming parts above or under the SLs) as in the $C_{p k}$ index in Kane [19].

Table 3 condenses the discussion of this section. The groups of multivariate PCIs according the approaches used in table 1 are compared through the criteria adopted in this section by using scores.

To sum up, on the one hand we can say that multivariate PCIs obtained through the derivation from univariate PCIs, as well as the ones based on the relation between PRs and SRs or the ones based on the PCA method try to describe the capability of multivariate processes in a global way (with a single index), but present weakness when estimating the proportion of nonconforming parts of the analyzed processes. On the other hand, multivariate PCIs obtained through the alternative definition for the $C_{p k}$ index proposed in Castagliola [64] deal with both topics, and thus, are more effective multivariate versions of the univariate PCIs. Nevertheless, these indices are calculated using high complex mathematical equations and furthermore, some of them present non robust behaviors because of the sampling using Monte Carlo.

\subsection{Selecting PCIs in capability analysis}

In this section we want to help the reader to understand how to use the information of this article to select PCIs for capability analysis. With this objective in mind, we use the flowchart in figure 16 . 


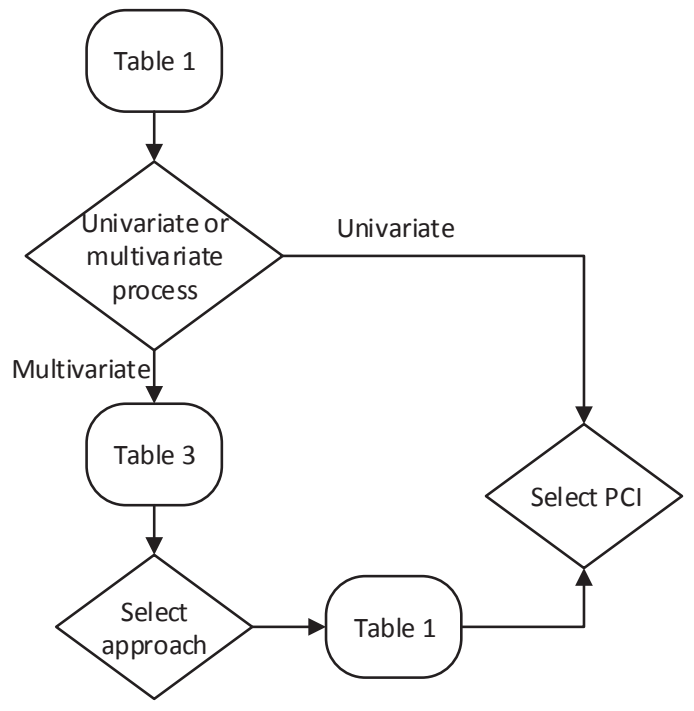

Fig. 16 Flowchart: Using tables 1 and 2 to select PCIs.

Starting with table 1 we suggest analyzing the data that we want to analyze in the capability analysis and distinguish between univariate and multivariate processes. In the case of univariate processes, we can select one PCI from table 1 by taking into account criteria such as the normality of the data or the information that is needed (output). In the case of multivariate processes, we need to go to table 3 and select one approach by taking into account the four criteria (calculation method, globality of the index, relation to NCP and robustness). Once the approach has been selected, we suggest going back to table 1 and select one multivariate PCI by taking into account criteria such as normality of the data or the information that is needed (output) .

\section{Conclusions and future lines of research}

In this article we classified and discussed univariate and multivariate PCIs from the literature, giving a useful and practical review to the industry. The review has been focused on univariate and multivariate PCIs based on the process capability definition introduced in the seminal paper of Kane [19].

All univariate and multivariate PCIs presented in this article are clustered in table 1 . To cluster the PCIs in table 1 , we defined some characteristics of the PCIs from the literature and we grouped them in three groups: the first group gives information of the process data; the second group describes the approach used to calculate the PCIs; and the third group is related to the information that the PCIs give.

The $C_{p k}$ index, which is an univariate $\mathrm{PCI}$, is widely used in the industry. Thus, this PCI cannot describe the capability of multivariate production processes. Thanks to multivariate PCIs, the correlation between product characteristics, which derives from the multivariate case, can be taken into account. Thus, the capability of production processes with multiple product characteristics that must fulfill simultaneously several requirements can be described with a single index. When analyzing the capability of multivariate production processes, univariate $\mathrm{PCIs}$ must be complemented with multivariate PCIs.

Regarding multivariate PCIs, we have seen that it is possible to distinguish between four different kinds of approaches: derivation from univariate PCIs, elimination of the correlation through the usage of the PCA method, comparison between shapes, and usage of the cumulative distribution function. We have seen that multivariate PCIs obtained through the derivation from univariate PCIs, as well as the ones based on the relation between PRs and SRs or the ones based on the PCA method try to describe the capability of multivariate processes in a global way (with a single index), but present weakness when estimating the the proportion of nonconforming parts of the analyzed processes. Multivariate PCIs obtained through the alternative definition for the $C_{p k}$ index proposed in Castagliola [59] deal with both topics. Nevertheless, these indices are calculated using high complex mathematical equations and furthermore, some of them present non robust behaviors because of the usage of Monte Carlo simulations.

As it has been seen in section 4, all multivariate PCIs present strengths and weaknesses. Thus, when analyzing the capability of multivariate production processes, decision makers of the industry may choose one multivariate PCI among all the PCIs presented in this article depending on different capability criteria. In this sense, we wanted to help researchers and practitioners to understand how to select PCIs for capability analysis. With this objective in mind, we provide a framework to explain how to use the information in tables 1 and 3. to select PCIs depending on the characteristics of the monitored process.

On the one hand, the authors of this article suggest using multivariate PCIs obtained through the alternative definition for the $C_{p k}$ index proposed in Castagliola [59] such as the $M C_{p k}$ indices in Shiau et al. [65] or in de-Felipe et al. [68] when analyzing the capability of multivariate production processes, in which the estimation of the proportion of nonconforming parts is rated 
as crucial. On the other hand, all other multivariate PCIs presented in this article can be applicable in capability analysis of multivariate production processes in which a direct relation to the proportion of nonconforming parts is not needed.

Taking into account the above conclusions we propose several opportunities for future research work in this field: First, we propose extending beyond the three categories in table 1 providing a far more options that might be encountered. Second, we suggest using the multivariate PCIs existing in the literature in real life applications and comparing the results obtained. Third, we propose using multivariate PCIs in monitoring of multivariate production processes and in decision making. Thus, a clear and unequivocal capability criterion for the multivariate case must be defined while describing the capability of multivariate processes with a single index.

\section{References}

1. Lupo T (2015) The new Nino capability index for dynamic process capability analysis. Quality and Reliability Engineering International 31(2): 305-312. doi: 10.1002/qre.1589.

2. Lepore A, Palumbo B (2015) New insights into the decisional use of process capability indices via hypothesis testing. Quality and Reliability Engineering International 31(8): 1725-1741. doi: 10.1002/qre.1713.

3. Jose KK, Luke JA (2013) Comparing two process capability indices under balanced one-way random effect model. Quality and Reliability Engineering International 29(2): 165-172. doi: 10.1002/qre.1297.

4. Aslam M, Wu CW, Azam M, Jun CH (2014) Mixed acceptance sampling plans for product inspection using process capability index. Quality Engineering 26(4): 450-459.

5. Krolczyk JB, Krolczyk GM, Legutko S, Napiorkowski J, Hloch S, Foltys J, Tama E (2015) Material flow optimizationa case study in automotive industry. Tehniki vjesnik 22(6): 1447-1456.

6. Legutko S (2009) Development trends in machines operation maintenance. Eksploatacja i NiezawodnoscMaintenance and Reliability (2): 8-16.

7. Taguchi G, Chowdhury S, Wu Y (2004) Taguchis Quality Engineering Handbook. John Wiley and Sons.

8. Oborski P (2014) Developments in integration of advanced monitoring systems. The International Journal of Advanced Manufacturing Technology 75(9-12): 1613-1632. doi: $10.1007 / \mathrm{s} 00170-014-6123-x$.

9. Stavropoulos P, Papacharalampopoulos A, Vasiliadis E, Chryssolouris G (2016) Tool wear predictability estimation in milling based on multi-sensorial data. The International Journal of Advanced Manufacturing Technology 82(1-4): 509-521. doi: 10.1007/s00170-015-7317-6.

10. Ostasevicius V, Jurenas V, Augutis V, Gaidys R, Cesnavicius R, Kizauskiene L, Dundulis R (2016) Monitoring the condition of the cutting tool using self-powering wireless sensor technologies. The International Journal of Advanced Manufacturing Technology 1-15. doi: 10.1007/s00170-0168939-z.

11. Kotz S, Johnson NL, Hubele NF, Spiring F, Cheng S, Yeung A, Leung B, Rodriguez R, Bothe D, Lu MW, Rudy RJ,
Vannman K, Boyles RA, Ramberg JS (2002) Process capability indices: A review, 1992-2000. Discussions. Journal of Quality Technology 34(1): 2-53.

12. Wu CW, Pearn WL, Kotz S (2009) An overview of theory and practice on process capability indices for quality assurance. International journal of production economics 117(2): 338-359. doi: 10.1016/j.ijpe.2008.11.008.

13. Yum BJ, Kim KW (2011) A bibliography of the literature on process capability indices: $2000-2009$. Quality and Reliability Engineering International 27(3): 251-268. doi: 10.1002/qre.1115.

14. Pan JN, Li CI, Chen FY (2014) Evaluating Environmental Performance using New Process Capability Indices for Autocorrelated Data. Environmental Monitoring and Assessment, 186(10): 6369-6384. doi: 10.1007/s10661-0143861-z.

15. Pan JN, Huang WKC (2015) Developing new multivariate process capability indices for autocorrelated data. Quality and Reliability Engineering International 31(3): 431444. doi: 10.1002 /qre.1603.

16. Ebadi M, Amiri A (2012) Evaluation of process capability in multivariate simple linear profiles, Scientia Iranica 19(6): 1960-1968. doi: 10.1016/j.scient.2012.09.010.

17. Wang FK, Tamirat Y (2015) Process Yield for Multivariate Linear Profiles with Onesided Specification Limits. Quality and Reliability Engineering International. doi: 10.1002/qre.1834.

18. Guevara RD, Vargas JA (2016) Evaluation of process capability in multivariate nonlinear profiles. Journal of Statistical Computation and Simulation 86(12): 2411-2428. doi: 10.1080/00949655.2015.1112391

19. Kane VE (1986) Process capability indices. Journal of Quality Technology 18(1): 41-52.

20. Montgomery DC (1996). Introduction to statistical quality control. John Wiley and Sons.

21. Sullivan LP (1985) Letters. Quality Progress 18: 7-8.

22. Tsai CC, Chen CC (2006) Making decision to evaluate process capability index $\mathrm{Cp}$ with fuzzy numbers. The International Journal of Advanced Manufacturing Technology 30(3-4): 334-339. doi: 10.1007/s00170-005-0052-7.

23. Bothe DR (1999) Composite capability index for multiple product characteristics. Quality Engineering 12(2): 253258. doi: $10.1080 / 08982119908962582$.

24. Chan LK, Cheng SW, Spiring FA (1988) A new measure of process capability: Cpm. Journal of Quality Technology 20(3): 162-175. doi: 10.1108/02656710110396076.

25. Pearn WL, Kotz S, Johnson NL (1992) Distributional and inferential properties of process capability indices. Journal of Quality Technology 24(4): 216-231. doi: $10.1080 / 03610929808832139$.

26. González I, Sánchez I (2009) Capability indices and nonconforming proportion in univariate and multivariate processes. International Journal of Advanced Manufacturing Technology 44:1036-1050. doi: 10.1007/s00170-008-1907-5.

27. Veevers A (1998) Viability and capability indexes for multiresponse processes. Journal of Applied Statistics 25(4): 545-558. doi: 10.1080/02664769823016.

28. Johnson $\mathrm{T}$ (1992) The relationship of Cpm to squared error loss. Journal of Quality Technology 24(4): 211-215.

29. Tsui KL (1997) Interpretation of process capability indices and some alternatives. Quality engineering 9(4): 587596. doi: 10.1080/08982119708919080.

30. Pearn WL, Chang YC, Wu CW (2006) Measuring process performance based on expected loss with asymmetric tolerances. Journal of Applied Statistics 33(10): 1105-1120. doi: 10.1080/02664760600746871. 
31. Abdolshah M (2015) Measuring Loss-Based Process Capability Index and Its Generation with Fuzzy Numbers. Mathematical Problems in Engineering. doi: $10.1155 / 2015 / 217406$.

32. Eslamipoor R, Hosseininasab H (2016) A Modified Process Capability Index Using Loss Function Concept. Quality and Reliability Engineering International 32(2): 435442. doi: 10.1002 /qre.1761.

33. Zwick DS (1995) A hybrid method for fitting distribution data and its use in computing process capability indices. Quality Engineering 7(3): 601-613. doi: 10.1080/08982119508918806.

34. Yang J, Gang T, Cheng Y, Xie M (2015) Process capability indices based on the highest density interval. Quality and Reliability Engineering International 31(8): 1327-1335. doi: 10.1002/qre.1665.

35. Piña-Monarrez MR, OrtizYañez JF, RodrguezBorbn MI (2015) Nonnormal Capability Indices for the Weibull and Lognormal Distributions. Quality and Reliability Engineering International 32(4): 13211329. doi: 10.1002/qre.1832.

36. Hubele NF, Montgomery DC, Chih WH (1991). An application of statistical process control in jet-turbine engine component manufacturing. Quality Engineering 4(2): 197210. doi: $10.1080 / 08982119108918906$.

37. Plante RD (2001) Process capability: a criterion for optimizing multiple response product and process design. IIE Transactions 33(6): 497-509. doi: 10.1080/07408170108936849.

38. Ch'ng CK, Quah SH, Low HC (2005) Index $\mathrm{C}^{*} \mathrm{pm}$ in multiple response optimization. Quality Engineering 17(1): 165-171. doi: 10.1081/QEN-200029001.

39. Śiman M (2014) Multivariate process capability indices: a directional approach. Communications in Statistics-Theory and Methods 43(9): 1949-1955. doi: 10.1080/03610926.2012.677926.

40. Wang FK, Chen JC (1998) Capability index using principal components analysis. Quality Engineering 11(1): 21-27. doi: $10.1080 / 08982119808919208$.

41. Tong YL (2012) The multivariate normal distribution. Springer Science and Business Media.

42. Shinde RL, Khadse KG (2009) Multivariate process capability using principal component analysis. Quality and Reliability Engineering International 25(1): 69-77. doi: 10.1007/s10182-011-0156-3.

43. Wang FK, Du TCT (2000) Using principal component analysis in process performance for multivariate data. The International Journal of Management Science 28(2): 185194. doi: 10.1016/S0305-0483(99)00036-5.

44. Jackson JE (1980) Principal components and factors analysis: part I - principal components. Journal of Quality Technology 12(4): 201-213.

45. Wang $\mathrm{CH}$ (2005) Constructing multivariate process capability indices for short-run production. International Journal of Advanced Manufacturing Technology 26(11-12): 1306-1311. doi: 10.1007/s00170-004-2397-8.

46. Perakis M, Xekalaki E (2011) On the implementation of the principal component analysis-based approach in measuring process capability. Quality and Reliability Engineering International 28(4): 467-480. doi: 10.1002/qre.1260.

47. Tano I, Vännman K (2013) A multivariate process capability index based on the first principal component only. Quality and Reliability Engineering International 29(7): 987-1003. doi: 10.1002/qre.1451.

48. Dharmasena LS, Zeephongsekul P (2015) A new process capability index for multiple quality characteristics based on principal components. International Journal of Production Research 2015: 1-17. doi: 10.1080/00207543.2015.1091520.
49. Chen H (1994) A multivariate process capability index over a rectangular solid tolerance zone. Statistics Sinica 1994: 749-758.

50. Das N, Dwivedi PS (2013) Multivariate process capability index: a review and some results. Economic Quality Control 28(2): 151-166. doi: 10.1515/eqc-2013-0022.

51. Ciupke K (2015) Multivariate process capability vector based on onesided model. Quality and Reliability Engineering International 31(2): 313-327. doi: 10.1002/qre.1590.

52. Chan LK, Cheng SW, Spiring FA (1991) A multivariate measure of process capability. International Journal of Modelling and Simulation 11(1):1-6.

53. Taam W, Subbaiah P, Liddy JW (1993) A note on multivariate capability indices. Journal of Applied Statistics 20(3): 339-351. doi: 10.1080/02664769300000035.

54. Braun L (2001) New methods in multivariate statistical process control. Diskussionsbeiträge des Fachgebietes Unternehmensforschung. Stuttgart. Universität Hohenheim.

55. Shaoxi W, Mingxin W, Xiaoya F, Shengbing Z, Ru H (2013) A multivariate process capability index with a spatial coefficient. Journal of Semiconductors 34(2). doi: 10.1088/1674-4926/34/2/026001.

56. Pan J, Lee C (2010) New capability indices for evaluating the performance of multivariate manufacturing processes. Quality and Reliability Engineering International 26(1): 315. doi: $10.1002 /$ qre.1024.

57. Pan JN, Li CI, Shih WC (2016) New multivariate process capability indices for measuring the performance of multivariate processes subject to non-normal distributions. International Journal of Quality and Reliability Management 33(1): 42-61. doi: 10.1108/IJQRM-12-2013-0195.

58. Jalili M, Bashiri M, Amiri A (2012) A new multivariate process capability index under both unilateral and bilateral quality characteristics. Quality and Reliability Engineering International 28(8): 925-941. doi: 10.1002/qre.1284.

59. Castagliola P (1996) Evaluation of non-normal process capability indices using Burr's distributions. Quality Engineering 8(4): 587-593. doi: 10.1080/08982119608904669.

60. Shahriari H, Hubele NF, Lawrence FP (1995) A multivariate process capability vector. Industrial Engineering Research Conference Proceedings: Nashville, Tennessee. doi: 10.1080/08982110902873605.

61. Niavarani MR, Noorossana R, Abbasi B (2012) Three new multivariate process capability indices. Communications in Statistics-Theory and Methods 41(2): 341-356. doi: 10.1080/03610926.2010.526743.

62. Grau D (2007) Multivariate capability indices for processes with asymmetric tolerances. Quality Technology and Quantitative Management 4(4): 471-488. doi: 10.1080/16843703.2007.11673166.

63. Pearn WL, Shiau JJH, Tai YT, Li MY (2011) Capability assessment for processes with multiple characteristics: a generalization of the popular index Cpk. Quality and Reliability Engineering International 27(8): 1119-1129. doi: 11191129. DOI: $10.1002 /$ qre.1200.

64. Castagliola P, Garca Castellanos JV (2005) Capability indices dedicated to the two characteristics case. Quality technology and Quantitative Management 2(2): 201-220. doi: $10.1108 / 13552510810861969$.

65. Shiau J, Yen C, Pearn WL, Lee W (2013) Yield-related process capability indices for processes of multiple quality characteristics. Quality and Reliability Engineering International 29(4): 487-507. doi: 10.1002/qre.1397.

66. Abbasi B, Niaki STA (2010) Estimating process capability indices of multivariate nonnormal processes. The International Journal of Advanced Manufacturing Technology 50(5-8): 823-830. doi: 10.1007/s00170-010-2557-y. 
67. Gu K, Jia X, Liu H, You H (2015) Yieldbased capability index for evaluating the performance of multivariate manufacturing process. Quality and Reliability Engineering International 31(3): 419-430. doi: 10.1002/qre.1602.

68. de-Felipe D, Klee T, Folmer J, Benedito E, Vogel-Heuser V (2016) A multivariate process capability index that complies with industry requirements. Conference of IEEE Industrial Electronics Society (IECON) 2016.

69. Chatterjee M, Chakraborty AK (2014) Exact Relationship of C"pk with Proportion of Nonconformance and Some Other Properties of C"p (u, v). Quality and Reliability Engineering International 30(7): 1023-1034. doi: 10.1002 /qre.1530.

70. Dianda DF, Quaglino MB, Pagura JA (2015) Performance of Multivariate Process Capability Indices under Normal and NonNormal Distributions. Quality and Reliability Engineering International. doi: 10.1002/qre.1939. 